\title{
Design and Implementation of Socially In- telligent Agents providing Emotional and Cognitive Support
}

\author{
Ryota YAMADA *1 $\bullet$ Hiroshi NAKAJIMA*1 • Jong- Eun Roselyn LEE*2 • \\ Scott Brenner BRAVE *3 $\bullet$ Heidy MALDONADO *4 $\bullet$ Clifford Ivar NASS *2 • \\ Yasunori MORISHIMA *5
}

\begin{abstract}
We propose a software platform for applications based on socially intelligent agents (SIAs). SIAs are software agents which manifest social intelligence. Although there are many types of social intelligence, we focus on two types, social intelligence for providing emotional support and social intelligence for providing cognitive support. By applying these types of social intelligence, our SIAs are able to simulate human social behavior. We demonstrate how we created the SIAs and describe the conceptual architecture of our software platform. We also show the mechanism for generating the agents' social behavior, which makes our software platform unique. According to the media equation theory, we expect SIAs to benefit users. To examine the effects of SIAs, we designed and implemented two applications. We provide explanation about how SIAs function in these applications and discuss further implications of social intelligence.
\end{abstract}

Keywords: socially intelligent agent, social intelligence, emotional support, cognitive support, media equation

\section{Introduction}

Human-machine interaction has become a crucial part of everyday life. According to the media equation theory [1], people treat computers, television, and new media as real people and places. The media equation theory indicates that media experiences equal human experiences. There is no switch in the brain that is activated when media are present. Media can evoke emotional responses, demand attention, influence memories, and so on. The theory suggests that manifestation of social behavior in machines, when used appropriately, can have positive effects on people's experiences with machines. Based on the media equation theory, we designed and implemented a software platform for creating applications using virtual characters that simulated human social behavior. We call such virtual characters as "socially intelligent

*1 Core Technology Center, OMRON Corporation

*2 Department of Communication, Stanford University

*3 Baynote Inc.

*4 School of Education, Stanford University

*5 College of Liberal Arts (Psychology), International Christian University agents (SIAs)" and the software "Software Platform for Agent-human Collaborating Environment (SPACE)".

This paper consists of six sections. In section 2, we introduce our idea of an SIA. In section 3, we propose a software platform named "SPACE", which enables us to create applications using these SIAs. In sections 4 and 5, we showcase two types of SIAs and their applications. In section 6 , we discuss implications of social intelligence, and in section 7 , we provide conclusions.

\section{SIA: Socially Intelligent Agent}

An SIA is a type of software agent. Although there are various definitions of software agents [2] [3] [4] [5], the following four dimensions are essential to defining any software agent: (1) autonomy, (2) reactivity, (3) pro-activeness, and (4) social ability. Social ability is the ability to engage other components of a system, in which agents are operating, through some sort of communication and coordination. We focused on the social ability of software agents, and we enhanced this social ability to enable effective collabo- 
ration not only with other components but also with human users. The social ability of software agents can be improved when social intelligence is embedded in software agents. We refer to a software agent with social intelligence as an "SIA".

\subsection{Social Intelligence}

There are many different types of social intelligence. We focused on two types of social intelligence. One is social intelligence for providing emotional support for users, and the other is social intelligence for providing cognitive support for users.

\subsubsection{Emotional Support}

Emotional support is support that makes people feel better. Emotional support is expressed through a caring orientation, e.g., giving positive comments or showing sympathy through comments and facial expressions.

\subsubsection{Cognitive Support}

Cognitive support is support that helps people better understand given information. Cognitive support reduces cognitive load, e.g., emphasizing or highlighting important information or guiding users to effectively navigate through information.

\section{SPACE: Software Platform for Agent - human Collaborating Environment}

SPACE provides basic functions for creating multiagent systems, such as management and facilitation of communication between agents. Such functions have also been provided by other known agent programming environments such as AGENT0 [6] and Open Agent Architecture [7]. What makes SPACE unique from these agent environments is a mechanism for manifesting social intelligence. With two types of social intelligence, emotional and cognitive, our agents show social behaviors similar to humans' . SPACE allows software developers to create SIAbased applications with greater ease and efficiency.

In 3.1, we show the conceptual architecture of SPACE, which enables social interaction between SIAs. In 3.2, we briefly describe the implementation of SPACE and its software development kit (SDK).

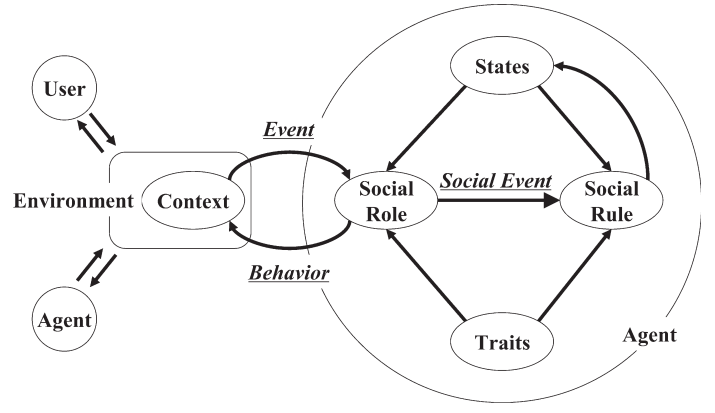

Fig.1 Conceptual Architecture of SPACE

\subsection{Conceptual Architecture of SPACE}

Figure 1 shows the conceptual architecture of SPACE. SPACE consists of a virtual environment and SIAs. In 3.1.1, we describe the environment, and in 3.1.2, we explain the agent.

\subsubsection{Virtual Environment}

The virtual environment, used for creating an application session using SPACE, contains information about the context of the application. According to this context, the virtual environment generates events and sends the information to the SIAs. The environment works as a hub for communication between agents. It enables the interaction between agents and maintains the context of interaction.

Context: The context includes the flow and the goal of the application. It also includes the information shared in the virtual environment. The context is designed to be application-specific.

\subsubsection{SIAs}

SIAs can be prepared to suit the demands of an application session based on SPACE. An SIA possesses traits, states, a social role, and social rules. These factors characterize each agent, which is autonomous. Some SIAs can represent users in a virtual environment. We call such SIAs as "avatars". Users can take part in the virtual environment via their own avatars.

Traits: A trait is static information, which does not change during an application session. Traits, which can be applied to each SIA, are designed to be independent from the applications.

States: A state is information that varies with each SIA. States, applied to each SIA initially, can be updated using social rules, which are described below. States are designed to be independent from the appli- 
cations.

Social Roles: Social roles are sets of rules for generating agent behavior with reference to their traits and states. The behaviors are explicit and implicit. These behaviors generate social effects for SIAs. We defined "social events" which cause social effects. Social events are designed to be abstract enough to be reused across different applications. They are used for processing social rules. Social events are sent to and processed by the SIA who generates them, and also by other agents via the virtual environment. Social roles are defined for each kind of role in each application using SPACE. Those agents who play the same kind of role in an application will play the same social role.

Social Rules: Social rules handle agents' states, traits and social events. Social rules are defined according to the theories of social science, including communication and social psychology. Social rules define how social events affect agents who send, receive, and observe social events. The idea that social events affect receivers and also senders and observers is important to the conceptual architecture of SPACE. Social rules are defined to be independent from applications. Those agents who work in SPACE use the same social rules. Social rules are abstract enough to be reused across different applications.

\subsection{Implementation of SPACE and its SDK}

We developed SPACE using Java. SPACE provides a server program, which runs virtual environments and SIAs. Virtual environments and SIAs are implemented as software agents, which interpret and process codes written in Lisp. SPACE also provides a set of codes for the virtual environment and SIAs, which are designed to be independent of applications. These codes include basic functions of the virtual environment, a variety of settings for traits that represents the personalities of SIAs, and social rules.

For creating an application based on SPACE, developers are required to prepare application-dependent portions of SPACE such as a context setting for a virtual environment, social roles and social status settings for SIAs, and a user interface (UI) program for users. To help prepare these portions, we provided SPACE software development kit (SDK). The SDK includes a scripting support tool, templates for SPACE portions and application programming interface (API) documents for developers.

Because software agents in SPACE run on processing codes written in Lisp, developers have to script portions, such as a context settings and social roles, in Lisp. To help developers who are not familiar with Lisp, we prepared a scripting support tool. The support tool enables developers to script these portions using XML. The support tool provides a set of XML tags defined for scripting these portions and rules for converting XML scripts into Lisp codes based on XSL. We defined 65 XML tags, which include tags for general programming and tags for controlling agents.

For example, we have tags like "integer" and "string" for defining types of data as tags for general programming. We also have tags like "if" and "loop" for programming. Here is an example of a code using XML tags for general programming:

$\langle$ if $\rangle$

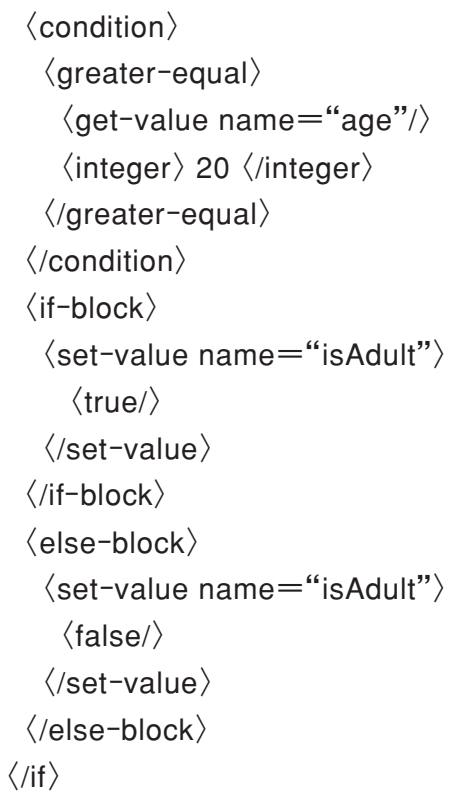

This code is translated into the following Lisp code by using the scripting support tool:

$$
\begin{aligned}
& \text { (if }(>=\text { age } 20) \\
& \quad(\text { setf isAdult } \mathrm{t} \text { ) (setf isAdult nil)) }
\end{aligned}
$$

Tags for controlling agents include "query", "wait", "social-event", and so on. Developers can define the agents' behavior by writing code using these tags. The use of these tags is explained with samples in the documentation included in the SDK.

The template set includes templates for the XML scripts and for UI programs. UI programs for SPACE 
may appear different depending on the applications. However, the basic role of the UI can be considered as a viewer for what is happening in the virtual environment, and the basic functions of the viewer, such as communicating between the virtual environment and SIAs in the virtual environment, should be common across all applications. Based on this idea, we call UI programs for SPACE as "environment viewers", and we prepared templates for creating them in Java. The templates are sets of sample codes, which can be used by developers as a starting point for programming. These templates provide the base of the code by default. Developers can then customize the templates and prepare their own code by editing them.

\section{SIA for Emotional Support based on SPACE}

To create an SIA for emotional support, we enabled social behaviors such as exchanging social responses by showing emotion based on facial expressions, giving verbal feedback, and so on. To manage the internal states of agents including emotion and the proper manifestation of the emotion, we designed a social model named "Interaction-X". In 4.1, we show how we configured SPACE for implementing SIAs for emotional support. In 4.2 , we describe details on Interaction-X, and in 4.3, we introduce an application of SIA for emotional support named "eSchool".

\subsection{Configuration of SPACE for Emotional Support}

To create SIA with emotional support based on SPACE, we configured the virtual environment and SIAs as follows:

\subsubsection{Virtual Environment for Emotional Support}

To create SIAs with emotional support, we set the virtual environment as a virtual space for collaboration between virtual characters. In this virtual space, users are represented as a virtual character.

Context: The context includes the flow and status of the virtual environment. For example, if the application is an eLearning application, the context includes the structure of lessons and how to run/manage them, and which step the virtual characters are in. The context also includes shared information. In the eLearning application, the context includes answers from students in the class.

\subsubsection{SIAs for Emotional Support}

SIAs for emotional support are virtual characters. The SIAs are not only autonomous agents, but also avatars controlled by the users.

Traits: The traits include the personality and social status of the SIA.

States: The states include emotions and desires of each SIA.

Social Roles: The social roles include rules for generating the behaviors of these virtual characters. These are explicit, such as saying something and showing facial expressions, or implicit, such as creating social atmospheres. This same eLearning example involves social roles such as teachers and students.

Social Rules: The social rules define how to update states when virtual characters send, receive, and observe feedback concerning tasks performed by other characters. For example, these rules can be used when agents, as teachers and students, exchange feedback about answers in the eLearning application.

\subsection{Interaction-X}

Interaction- $\mathrm{X}$ was designed based on theories and research in the field of social science such as the OCEAN five dimension personality model [8], appraisal theory [9], empathy [10], interpersonal attraction [11], and self-efficacy [12].

Based on the OCEAN five dimension personality model, we used the following five traits for personality: openness to experience (intellect), conscientiousness, extraversion, agreeableness, and neuroticism (emotional stability).

The appraisal theory states that emotion is essentially a reaction to events deemed relevant to the needs and goals of an individual. Based on this theory, we used an event model for Interaction-X.

The theories on empathy, interpersonal attraction, and self-efficacy were used for designing a formula for maintaining the states of SIAs.

We designed Interaction- $\mathrm{X}$ as an abstract and reusable model for social behaviors, and we developed it as a component of SPACE. In SPACE, Interaction$\mathrm{X}$ is an implementation of social rules. In configuring SPACE for emotional support, the emotions of each agent were included in states as we mentioned in 4.1.2. Currently, we represent emotions using continuous variables. We also use Ekman's six basic emotions 


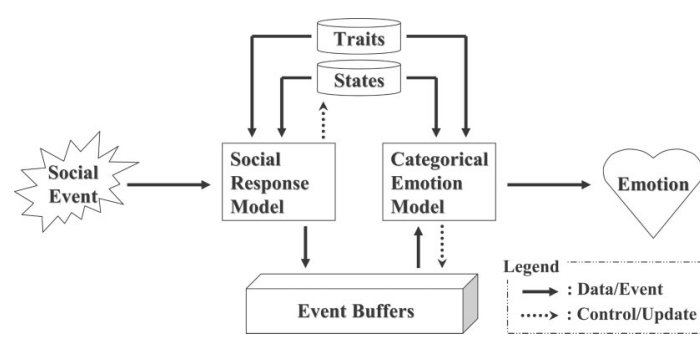

Fig.2 Outline of Interaction $-\mathrm{X}$

[13] for expressing agents' emotion with facial expressions. Our system provides seven types of emotions, neutral and the six basic emotions: happy, sad, angry, fear, disgust, and surprise. As happy, sad, and angry has three levels for each, and fear has two levels, our system provides 14 discrete emotions. We call these 14 discrete emotions as "categorical emotions". We use these 14 emotions because they are considered universally recognizable across cultures. Interaction-X can be considered as a mechanism for maintaining agents' states and generating their categorical emotions from social events.

Figure 2 shows the outline of Interaction-X. Interaction- $X$ consists of a social response model and a categorical emotion model.

\subsubsection{Social Response Model}

The social response model is a mechanism for maintaining agents' states, traits, and social events. Figure 3 illustrates the social response model.

Traits consist of intelligence, conscientiousness, extraversion, agreeableness, emotional stability, and social status values for every agent in the virtual environment. States of each agent consist of emotion, confidence, liking values for every agent in the virtual environment, a desired behavior buffer for maintaining desire, and event buffers for maintaining emotional events (short-lived emotional event buffer and mo-

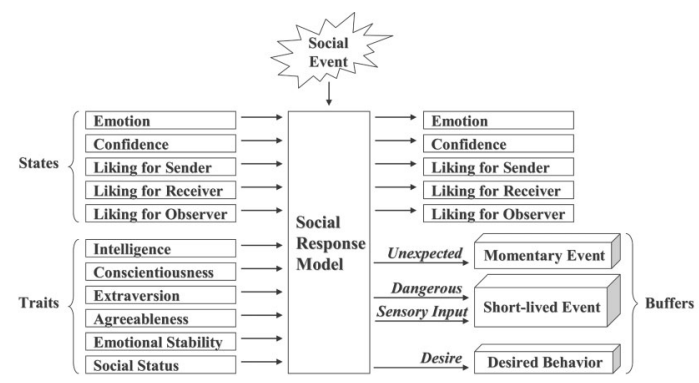

Fig.3 Social Response Model mentary emotional event buffer). These states are maintained when each agent receives a social event from the virtual environment. Currently, we use three kinds of social events: task request, task feedback, and social feedback. Each event consists of an event identifier, source, destination, and degree. The social response model has a set of rules for handling each event. Rules for each event consist of rules for senders, receivers, and observers. Each rule for specific agents describes how to update values in states and how to put/remove desires and emotional events into/ from buffers in the states. Developers can use each value in the states and traits for specifying agent behavior. Each desire stored in the desired behavior buffer is used for triggering specific agent behavior. We use desires for giving social feedback. Since social response model maintain these values and desired behavior buffers, it is not necessary for developers to know how these values and buffers should be maintained. Each emotional event stored in the event buffer is used in the categorical emotion model described below. We employ sensory input, dangerous, and unexpected events. Sensory input and dangerous events are maintained in the short-lived emotional event buffer, and an unexpected event is maintained in the momentary emotional event buffer.

\subsubsection{Categorical Emotion Model}

The categorical emotion model is a mechanism for generating categorical emotions reflecting agents' states, traits, and event buffers. Figure 4 illustrates the categorical emotion model.

With the categorical emotion model, we classified seven types of emotions in three different categories: lasting, short-lived, and momentary emotions. Lasting emotions consist happy, sad, angry, and neutral. These emotions are derived from the emotion value in states and agreeableness value in the traits. Short-

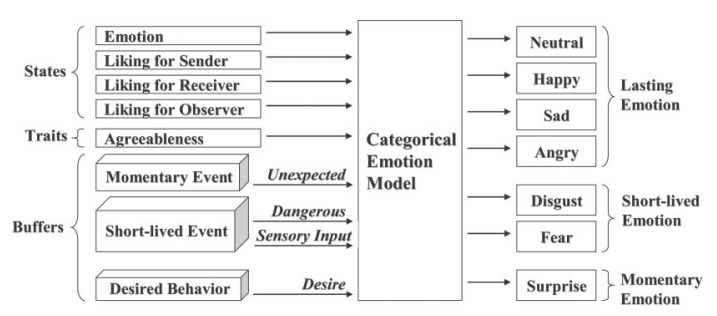

Fig.4 Categorical Emotion Model 
lived emotions consist of fear and disgust. These emotions are derived from the liking values in the states and emotional events in the short-lived emotional event buffer. The momentary emotion consists of surprise. These emotions are derived from the emotional events in the momentary emotional event buffer. The emotions in the category of lasting emotions are emotions that continue successively based on events and emotions in last moment. The emotions in the short-lived emotions category are emotions that last while the sources of the emotions remain, and they are shown in prior to lasting emotions. The emotions in the momentary emotions category are the emotions that are expressed shortly after the source of the emotions emerges, and they are shown in prior to short-lived and lasting emotions. According to these rules, agents are able to resolve the conflict between these emotions.

\subsection{Application of SIA for Emotional Support}

To study the effects of SIAs for Emotional Support, we designed an application named "eSchool" for eLearning using SPACE. In 4.3.1, we show the settings of eSchool. In 4.3.2, we explain how SIAs work in eSchool.

\subsubsection{What is eSchool?}

eSchool is a collaborative learning system that provides a virtual space in which students can study anytime. Figure 5 shows a screenshot of eSchool, which uses a classroom setting.

In the classroom, there are two students and one teacher. A user is represented by a student avatar.

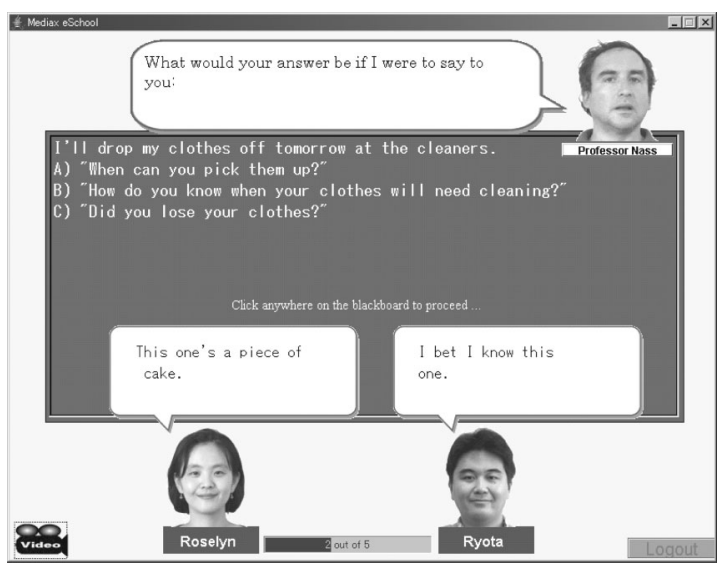

Fig.5 Screenshot of eSchool
The teacher and co-learner classmate are autonomous agents. These avatars and agents are SPACE based SIAs. In figure 5 , the user's avatar is shown in the lower left. The teacher is in the upper right, and the classmate is in the lower right. The area in the middle of the classroom represents a blackboard. In the classroom, the teacher gives the students lessons and quizzes, and the classmate and the teacher provide social support and engagement for the user. The eSchool application tracks the progress of the user over multiple sessions, and provides multimedia support such as video content. Currently, eSchool is being used for English instruction for non-native speakers. However, it can be adapted to any learning context.

\subsubsection{How SIAs Work in eSchool}

In eSchool, the avatar, the classmate, and the teacher show social behaviors relevant to the given situation. In each step, events are distributed to all SIAs (the avatar, the classmate, and the teacher). Each SIA reacts to the events based on Interaction$\mathrm{X}$. Here are some examples of social behaviors:

Expressing confidence: If the avatar and the classmate are confident, they express their confidence when the teacher gives a quiz. Figure 5 is an example of this situation. After the teacher gives a quiz item, the students (i.e., the user avatar and the classmate) choose their answer, and then the teacher calls on a student to give her/his answer to the class. That student expresses her/his confidence based on Interaction-X. When the system goes into the teacher giving a quiz, the events are distributed to the students. If a student is confident enough, she/he will become more positive and change her/his facial expression to show happiness. Each student reacts to the distributed event. The event includes parameters such as sender, receiver, and degree of difficulty of the quiz. Each student updates the emotional value and confidence in regards to the agent's states, traits, and parameters of the event. For example the emotional value of a student will be updated in regards to the following values: the emotion before receiving the event, confidence before receiving the event, the emotional stability included in the traits, conscientiousness included in the traits, and so on. After the emotion value is updated, the facial expressions displayed on the screen are updated. Each SIA handles its state, 
traits, and buffers based on Interaction-X. For example, if the emotional value is greater than the defined threshold and there are no events stored in the buffers, the SIA changes the facial expression to happy. If the emotional value is less than the defined threshold and there are no events stored in the buffers, the facial expression will be sad or angry. Whether the SIA manifests sadness or anger will be decided by the agreeableness value in traits. On the other hand, if the student is not confident enough, she/he will become more negative and change her/his facial expression to be sadder or angrier, and/or answer with statements which indicate a lack of confidence such as "Maybe ..." and "Um...". If the confidence value is greater or less than the defined threshold, the avatar or the student will show confidence. Whether a student shows confidence or not is decided by looking at the extraversion value. The extraversion value is a trait of SIA given as a real value between 0 and 1 . This value is used as the likelihood for making manifestation.

Responding to feedback: After the student answer the teacher, the teacher will give feedback on whether the answer is correct or not. The student will become either more positive or more negative depending on if the answer was correct or incorrect. If the answer is incorrect when the student is confident enough, they will be surprised. The student who was not called on by the teacher will also become more positive or more negative based on their personality (e.g., agreeable).

Providing social feedback: After the teacher gives feedback on the answer, the teacher can add social feedback comments such as "Good job" or "Nice try". The student who was not called on by the teacher can also provide social feedback such as "That was hard, and you got it!", "Lucky guess!", and "Ha, ha, you got it wrong!”. Whether each agent gives social feedback or not and what kind of comment she/he makes are determined by the state of emotional value, liking, and personality (e.g., how extroverted she/he is).

\section{SIA for Cognitive Support based on SPACE}

To create an SIA for cognitive support, we enabled social behaviors such as properly drawing attention of user in the eLearning system's UI by pointing out important information, hiding comparatively unimportant information, and so on. To properly manage agents' behavior, we designed a social model named "Expression-X". In 5.1, we show how we configured SPACE for implementing SIAs for cognitive support. In 5.2, we describe the details of Expression-X. In 5.3, we introduce an application for SIAs for cognitive support named "eNavi".

\subsection{Configuration of SPACE for Cognitive Support}

To create a SPACE based SIA for cognitive support, we configured the virtual environment and SIAs as follows:

\subsubsection{Virtual Environment for Cognitive Support}

To enable SIAs for cognitive support, we set the virtual environment as a virtual space for collaboration between SIAs as components of a type of UI software. In the virtual space, SIAs as UI components, such as buttons, labels, panels, and tables were included.

Context: The context includes the flow and status of the virtual environment. For example, if the application is a type of UI software, the context includes the status of the running software, and which step users are in. The context also includes the input history from users. For example, in diagnostic software UI, the context includes diagnostic answers from users.

\subsubsection{SIAs for Cognitive Support}

Each component included in the UI is an SIA for cognitive support. The SIAs include not only general components such as buttons, labels, panels, and tables, but also virtual 3D characters which can show some gestures.

Traits and States: Properties of each component SIA, including color, size, and position, are either traits or states. If the value of a property is fixed, it will be a trait. If the value of a property is variable, it will be a state. In addition to general properties, component SIAs have relational information and a priority value as additional properties. This information is used to control SIA behavior.

Social Roles: The social roles include rules for generating SIA behaviors regarding states. The behaviors include general behaviors of UI components such as showing and/or hiding information, and receiving 
input from users. The behaviors also include actions of virtual 3D characters such as moving, pointing to a particular point on the screen, and using certain gestures. For example, if the application is a UI for diagnostic software based on Q\&A session between the system and user, it will involve social roles such as a question-displaying component and an answer-receiving component.

Social Rules: The social rules define how to update states of SIAs when the status of virtual space changes. For example, when the status of software changes, the priority value of each component SIA will be updated based on the social rules.

\subsection{Expression-X}

Expression- $\mathrm{X}$ was designed based on the theories and research in the area of social science such as source credibility [14], distributed cognition [15] [16], and kinesics [17].

The theory of source credibility states that people are more likely to be influenced by the source when it is presented and perceived as credible. To improve the credibility of each component SIA, we provide a framework for giving SIAs specific roles. Based on a given role, the behavior of each SIA is guided to be consistent. This framework is based on the social roles in SPACE. By providing the framework, we prevent developers from implementing UIs that includes inconsistent components.

In the distributed cognition theory, cognition and intelligence are viewed as collaborative and interactive processes and products between people and various agents and artifacts in the environment. To improve cognition, we provided a mechanism for component SIAs working collaboratively. This mechanism is based on the conceptual architecture of SPACE. Based on this mechanism, events, such as inputs from the user and changes of the state of the system, will be delivered not only to specific SIAs involved with the event but also to all the other SIAs in the system.

The kinesics theory states that body languages, such as eye contact and gestures, are communicative and convey social meanings, such as power, intimacy, liking, social distance, and confidence. Based on this theory, we designed the behavior of SIAs into virtual $3 \mathrm{D}$ characters.

We designed Expression- $\mathrm{X}$ as an abstract and re- usable model for social behaviors. In SPACE, Expression- $\mathrm{X}$ is an implementation of social rules. In configuring SPACE for cognitive support, variable properties of each component SIA were included in states as we mentioned in 5.1.2. The properties and behavior of the general components of a UI are limited and common. On the other hand, additional properties such as relational information and priority are added to the configuration, and virtual $3 \mathrm{D}$ characters are included as UI components. In Expression-X, we define how to handle additional properties. We also define basic properties and behavior of the virtual 3D characters for drawing the attention of users. Expression-X can be considered as a mechanism for maintaining agents' states and a guideline for creating UIs using SIAs.

\subsubsection{Handling Additional Properties}

Based on the mechanism of SPACE, events generated in the virtual environment are distributed to every SIA. According to this mechanism, every component SIA receives events. Each SIA updates its states based on social rules reflecting its own traits and states. At that time, additional properties of SIA, such as relational information and priority, play significant roles.

Relational Information: Relational information defines the relationship between component SIAs. Each component has a parent and a child. There must be one parent for each component. However, the number of children can vary. Each component can check the state of parent component. This is used to maintain the priority value. Each component reacts to events after its parent finishes reacting to the event.

Priority: Priority is a value for determining whether each component SIA should be shown or hidden. If the priority value is greater than the defined threshold, the component will be shown. If it is less than the defined threshold, the component will be hidden. The value of the threshold should be defined as part of the social role. This is because whether each component should be shown or hidden is supposed to be strongly related to each application. In the UI, some components are located on others. In this situation, if a component is shown or hidden, other components located on the component should be shown or hidden at the same time. To actualize this behavior, par- 


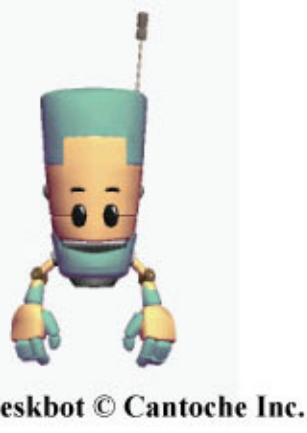

Fig.6 Example of a Virtual 3D Character

ents always react sooner than the children as described before. Then, when the children react to the events, they can check the priority value from their parents and overwrite their own priority value if it is necessary.

\subsubsection{Basic Properties and Behaviors of Virtual 3D Characters for Drawing Attention}

We defined position, size, direction of pointing, and pointing method as basic properties of virtual 3D characters. We selected the pointing gestures animated by Living Actor ${ }^{\mathrm{TM}}$ technology to display varying degrees of cognitive feedback.

Position: Position indicates where the virtual 3D character is located on the screen. The value is a combination of integers which indicates the position on the $\mathrm{X}$ - and $\mathrm{Y}$-axes.

Size: Size indicates how big the virtual 3D character is on the screen. The value will be the percentage of image scaling.

Direction of Pointing: Direction of pointing indicates which direction the virtual character points. Currently, there are ten values: top, bottom, left, right, upper left, upper light, lower left, lower right, back, and front.

Pointing Strength: Pointing strength indicates how strong the virtual 3D character should draw attention of the users by pointing. Currently, we use a virtual $3 \mathrm{D}$ character with a head and two hands. Figure 6 is an example of a virtual 3D character [18]. This character can use line of sight and a finger to draw attention. We use three levels of strength.

From the basic properties shown above, virtual 3D characters can move and point.

Move: From the position and size values, virtual 3D
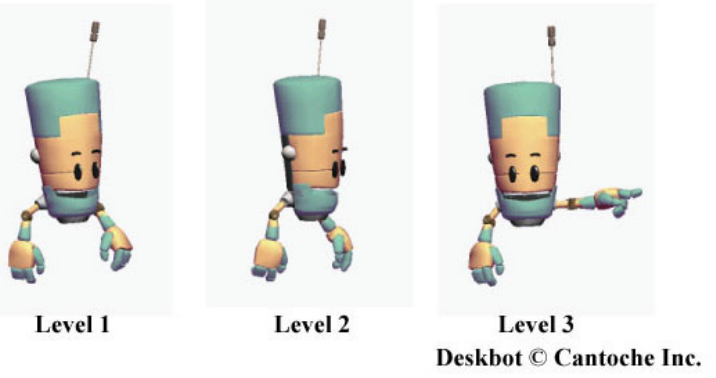

Fig.7 Examples of a Virtual Character Pointing

characters move on the screen. If the position value changes, the character will move from its current position to a given position, and the size of character will change. It will be displayed as if the character stepped back or forward on the screen.

Point: From the direction of pointing and pointing strength values, virtual 3D characters point on the screen. Figure 7 shows examples of a virtual character pointing. The character on the left is pointing to the right using only a line of sight (Level 1 ). The center character is pointing right with line of sight and body movement (Level 2). The character on the right is pointing right with a finger (Level 3).

In addition to the behaviors above, these virtual characters also show text messages using speech balloons.

\subsection{Application of SIA for Cognitive Support}

To study the effects of SIAs for Cognitive Support, we designed and implemented an application named "eNavi". In 5.3.1, we show the settings of eNavi, and in 5.3.2, we explain how SIAs work in eNavi.

\subsubsection{What is eNavi?}

eNavi is a UI for an eLearning system based on Q\&A sessions between the system and a user. We attached a UI using SIAs to Q\&A based software for eLearning. The software provides questions for users. Then, the users are required to answer the question. After users answer the question, the correct answer and explanation are shown. Some words shown in the explanation may be difficult for users. To support users' understanding, the software provides hyperlinks for key terms. When users click a hyperlink, an explanation of the word is shown. When users learn with the software, users are able to see the questions, the cor- 


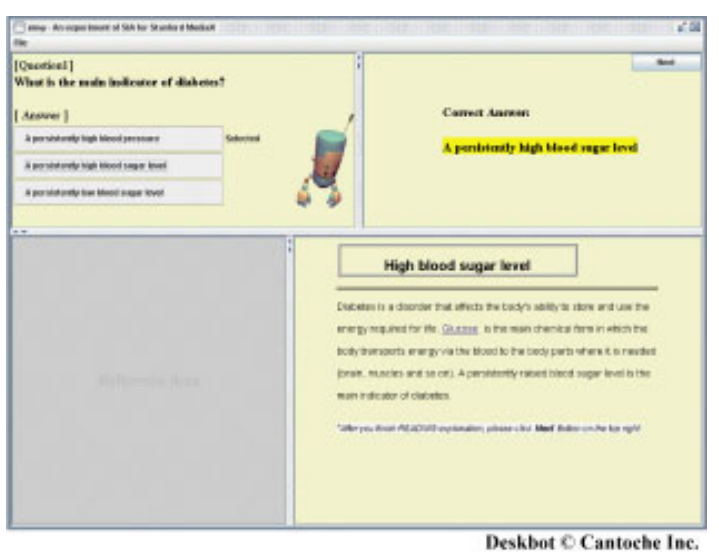

Fig.8 Screenshot of eNavi

rect answers, explanations of the answers, and explanation of the key terms. There is a great amount of information shown on a screen, and if users are not familiar with the software, they may become confused and even become frustrated. The objective of eNavi is to reduce this confusion or frustration. Figure 8 shows a sample image of eNavi. The UI is divided into four sections and with one virtual character. The upper left section is for displaying questions. The upper right section is for showing the correct answer. The lower right section is for showing the explanation of the answer. Finally, the lower left section is for showing the explanation of key terms. The location of these sections is designed to make users follow clockwise as they engage in the learning process.

\subsubsection{How SIAs work in eNavi}

In eNavi, each component in the UI is an SIA. When users learn with the software, each SIA works to re-

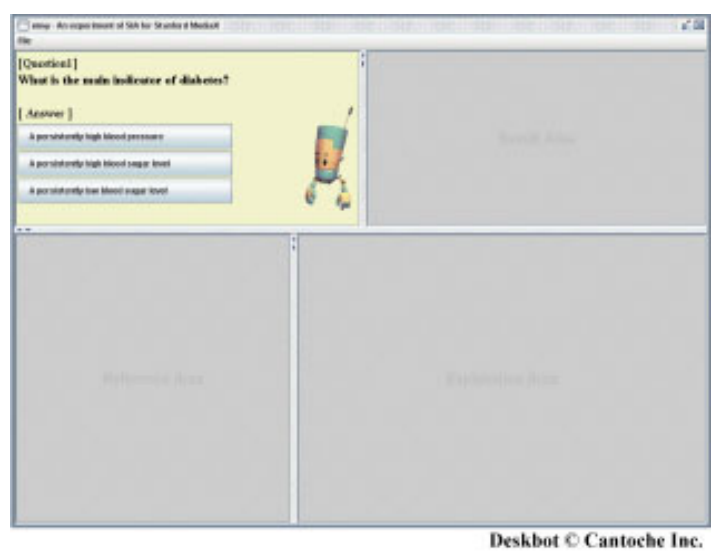

Fig.9 Showing Question duce the cognitive load of users by showing or hiding itself. In each step, events are distributed to all SIAs, and each SIA reacts to the events based on Expression-X.

When the system displays a question, only components in the question section and the virtual character (tutor agent) is shown. At that moment, the virtual character draws attention of user to the question using line of sight. Figure 9 is an example of this situation. When the system goes into the step for displaying a question, events indicating the step are distributed to all SIAs. As reaction to the events, each SIA behaves as follows: An SIA in the question section show itself. The SIAs in the correct answer, the answer explanation, the key-term explanation sections hide themselves, and the virtual 3D character uses line of sight to draw attention.

After reading a question, users choose an answer. Then, the correct answer key will be shown. The virtual character then guides users using text messages in balloon.

After showing the correct answer, additional information for the Q\&A is shown. At that time, the virtual character guides users, and draw their attention to the answer explanation using a finger.

If users click on the hyperlinks in the additional information section, related explanation will be provided. At that moment, the virtual character draws attention of users to the explanation section using line of sight with body movement. Figure 10 shows an example of this situation.

In each step, events are distributed to all SIAs. Then, each SIA decides its behavior based on the

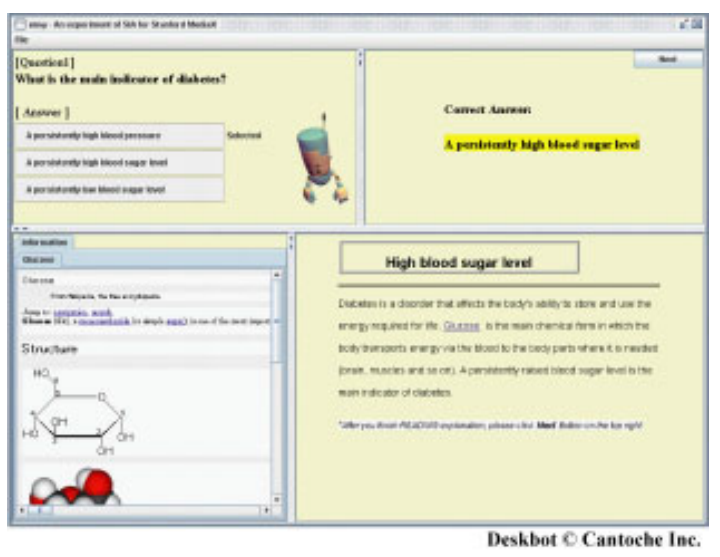

Fig.10 Showing Explanation 
mechanism of SPACE and rules in Expression-X. Although it is desirable to configure parameters in Expression-X such as thresholds for behaviors to optimize SIAs, SPACE and Expression- $\mathrm{X}$ are expected to be reusable as a guideline for creating socially intelligent UIs based on SIAs.

\section{Discussion}

As we described above, we designed and implemented SIAs for emotional and cognitive support. We expect that SIAs with context-appropriate social behaviors shown above will have positive effects on users. To substantiate our prediction, we conducted experiments. The results of our experiments show that just as social behaviors have positive effects on trust and learning in human-human interactions, social behaviors manifested by SIAs also showed positive effects.

For example, we conducted an experiment for examining social behaviors of SIAs using eSchool. A total of 77 undergraduate students in Japan participated in the experiment.

We prepared three versions of eSchool as follows: eSchool with no agent (No-Agent condition), eSchool with agents without social behavior (Agent condition), and eSchool with agents with social behavior (SIA condition). After participants used eSchool, a short quiz followed to measure the participants' performances. Table 1 shows these results.

In Table 1, the values in "Correct" column indicate the percentage of correct answered and the values in "No Answer" column indicate the percentage of questions not answered. The results show that participants who were in SIA condition performed better and answered more questions than in Agent or No-Agent conditions. These results indicate that social behavior provides positive effects for performance.

For more detailed information about our experiments, please see [19] [20] [21] [22] [23].

In section 4 , we presented software that uses social

Table 1 Short Quiz Results

\begin{tabular}{|r|l|r|r|}
\hline \multicolumn{2}{|c|}{} & Correct & No Answer \\
\hline \multirow{3}{*}{ Conditions } & No-Agent & $51 \%$ & $27 \%$ \\
\cline { 2 - 4 } & Agent & $60 \%$ & $22 \%$ \\
\cline { 2 - 4 } & SIA & $69 \%$ & $7 \%$ \\
\hline
\end{tabular}

intelligence, which manifests emotional support. More specifically, our agents showed social behavior in emotional reaction appropriate to the given context. The most interesting point was that the social behavior of agents had positive effects not only on the emotion of the user, but also on her/his performance. While the social behavior manifested in the application successfully provided emotional support, there are different types of social behavior for other emotional support. For example, agents can change their behavior based on the emotional states of the users expressed by their avatars. Different types of social behavior for emotional support can be used in combined forms. However, we will need to conduct further research regarding how to combine and manage multiple social behaviors.

In section 5, we presented software that uses social intelligence, which manifests cognitive support. More specifically, our agents showed social behavior in cognitive navigation appropriate to the context. To make the navigation process work, we focused on two points. One was to reduce the cognitive load for users by limiting displayed information. The other was to support users keeping by maintaining consistency by assigning specific roles and properly consistent behaviors to each component in the UI.

We introduced emotional and cognitive support separately. However, we expect that emotional and cognitive support can provide more positive effects when they are combined. In future research, we hope to effectively integrate social intelligence for emotional and cognitive support.

\section{Conclusion}

We proposed a software platform for applications using socially intelligent agents (SIAs). Although there are many types of social intelligence, we focused on two types, social intelligence for providing emotional support, and social intelligence for providing cognitive support. By applying these two types of social intelligence, our SIAs showed human-like behavior. According to the media equation theory, we expect SIAs will have beneficial effects for users. To explore this possibility, we implemented two applications of our SIAs. Our experiments showed that SIAs are beneficial not only for users' emotions but also for their performances. 


\section{Acknowledgements}

We would like to express our thanks to Kimihiko Iwamura, Ritsuko Niside, Wataru Hasegawa, and Omron Advanced Systems, Inc. for helping with the collaboration between OMRON Corporation and Media-X at Stanford University. We would also like to thank Keiko Hayashi and Yuriko Iwashita for helping with the implementation of SPACE and its applications, and Benoit Morel, Laurent Ach, and Cantoche Inc. for providing the Living Actor ${ }^{\mathrm{TM}}$ technology, which animated the virtual 3D character Deskbot, for our study.

\section{References}

[1] Byron Reeves and Clifford Nass, "The Media Equation: How People Treat Computers, Television, and New Media Like Real People and Places", Stanford University Center for Study of Language and Information, 1996.

[ 2 ] Hyacinth S. Nwana, "Software Agents: An Overview", Knowledge Engineering Review, Vol.11, No.3, pp.140, Cambridge University Press, Sep. 1996.

[ 3 ] Gerhard Weiss, "Multiagent Systems: A Modern Approach to Distributed Artificial Intelligence", MIT Press, 1999.

[ 4 ] Michael Wooldridge, "An Introduction to Multiagent Systems", John Wiley \& Sons, Feb. 2002.

[ 5 ] Stuart Russell and Peter Norvig, "Artificial Intelligence: A Modern Approach (Second Edition)", Prentice Hall, 2002.

[6] Yoav Shoham, "Agent0: A Simple Agent Language and its Interpreter", Proceedings of the Ninth $\mathrm{Na}$ tional Conference on Artificial Intelligence (AAAI91, pp.704- 709, 1991.

[ 7 ] Philip R. Cohen, Adam Cheyer, Michelle Wang, and Soon Choel Baeg, "An Open Agent Architecture", Proceedings of AAAI Spring Symposium, pp.1-8, Mar., 1994.

[ 8 ] Jannica Heinstrom,"Five Personality Dimensions and Their Influence on Information Behaviour”, Information Research, 9(1), Oct. 2003.

[9] I. J. Roseman, A. A. Antoniou, and P. E. Jose, "Apprisal Determinants of Emotions: Constructing a More Accurate and Comprehensive Theory", Cognitive and Emotion, 10 (3), pp.241-277, 1996.

[10] C. D. Batson, C. L. Turk, L. L. Shaw, and T. R. Klein, "Information Function of Empathic Emotion: Learning that We Value the Other's Welfare", Journal of Personality and Social Psychology, 68 (2), pp.300-313, 1995.

[11] R. C. Curtice and K. Miller, "Believing Another Likes or Dislikes You: Behaviors Making the Beliefs Come True", Journal of Personality and Social Psychology, 51, pp.284- 290, 1958.

[12] Howard S. Friedman, Nancy E. Adler, Ross D. Parke,
Christopher Peterson, Robert Rosenthal, Ralf Schwarzer, Roxane C. Silver, and David M. D. Spiegel, "Encyclopedia of Mental Health", Academic Press, 1998.

[13] Paul Ekman and Wallance V. Friesen,"Unmasking the Face: A Guide to Recognizing Emotions from Facial Expressions", Malor Books, Cambridge, MA, 2003.

[14] Carl I. Hovland, Irving L. Janis, and Harold H. Kelley, "Communication and Persuasion", New Haven, CT: Yale University Press, 1953.

[15] Edwin Hutchins, "Cognition in the Wild", Bradford Books, 1995.

[16] Mark Perry, "Distributed Cognition”, In John M. Carroll(Ed. "HCI Models, Theories, and Frameworks: Toward a Multidisciplinary Science", Morgan Kaufmann, 2003.

[17] Ray L. Birdwhistell, "Kinesics and Context: Essays on Body Motion Communication”, University of Pennsylvania Press, 1970.

[18] Benoit Morel, "Recruiting a Virtual Employee : Adaptive and Personalized Agents in Corporate Communication," In Sabine Payr \& Robert Trappl (Eds) "Agent Culture", Lawrence Erlbaum, 2004.

[19] Jong-Eun Roselyn Lee, Cliff Nass, Scott Brave, Yasunori Morishima, Hiroshi Nakajima, and Ryota Yamada,"The case for caring co-learners: The effects of a computer-mediated co-learner agent on Trust and Learning", Journal of Communication, Vol.57, No.2, pp.183-204, Jun., 2007.

[20] Heidy Maldonado, Jong - Eun Roselyn Lee, Scott Brave, Cliff Nass, Hiroshi Nakajima, Ryota Yamada, Kimihiko Iwamura, and Yasunori Morishima, "We Learn Better Together: Enhancing eLearning with Emotional Characters", Proceedings of the Computer Supported Collaborative Learning 2005 (CSCL2005), Taipei, Taiwan, May 2005.

[21] Jong-Eun Roselyn Lee, Heidy Maldonado, Clifford I. Nass, Scott B. Brave, Ryota Yamada, Hiroshi Nakajima, and Kimihiko Iwamura, "Can "Cooperative" Agents Enhance Learning and User-Interface Relationship in Computer-based Learning Environment?”, Proceedings of the 2005 International Communication Association Conference (ICA2005), New York, US, May. 2005.

[22] Yasunori Morishima, Hiroshi Nakajima, Scott Brave, Ryota Yamada, Heidy Maldonado, Clifford Nass, and Shigeyasu Kawaji, "The Role of Affect and Sociality in the Agent-Based Collaborative Learning System", Proceedings of the Affective Dialogue Systems (ADS2004), pp.265-275, Jun. 2004.

[23] Hiroshi Nakajima, Yasunori Morishima, Ryota Yamada, Scott Brave, Heidy Maldonado, Clifford Nass, and Shigeyasu Kawaji,"Social Intelligence in a HumanMachine Collaboration System-Social Responses of Agents with Mind Model and Personality", Journal of Japanese Society for Artificial Intelligence(JSAI), Vol.19, No.3, 2004. (in Japanese) 
[Contact Address]

Core Technology Center,OMRON Corporation

9-1 Kizugawa-dai, Kizugawa-City

Kyoto 619-0283 JAPAN

Ryota YAMADA

TEL : + 81-774-74-2158

FAX : + 81-774-74-2004

E-mail : ryamada@ari.ncl.omron.co.jp

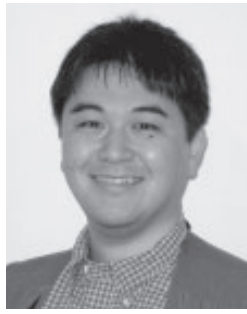

Ryota YAMADA [member]

Ryota Yamada received B. E. and M. E. degrees from Nagoya Institute of Technology in 2000 and 2002. He joined OMRON Corporation in 2002. $\mathrm{He}$ is currently a software engineer and a researcher at Core Technology Center of OMRON Corporation. He was a visiting researcher at Media-X at Stanford University from 2003 to 2006. His research interests include human-machine collaboration and computer supported cooperative work.

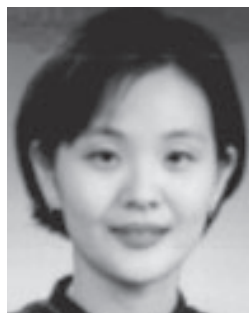

Jong - Eun Roselyn LEE [non-member]

Jong-Eun Roselyn Lee (MA, Seoul National University) is a Ph.D. candidate in Communication at Stanford University and a member of the Communication between Humans and Interactive Media(CHIMe) Lab directed by Dr. Clifford Nass. She has conducted research on effects of embodied agents on users' perception of and relationship with computer interfaces; her research has also investigated how socially intelligent agents that manifest caring orientations toward users can enhance learning. Her dissertation research explores social identity dynamics in avatar-represented group interaction.

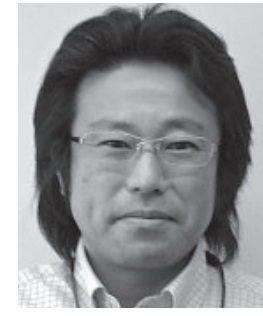

Hiroshi NAKAJIMA [member]

He recieved Ph.D. degrees from Kumamoto University, Japan, in 2004. $\mathrm{He}$ is a senior technology specialist of OMRON Corporation. His interests have been human-machine collaborative systems with optimal integration of human and machine intelligence based on fuzzy logic and soft computing. He is a member of IEEE, ACM, AAAI, JSKE, SOFT, JSAI, and IPSJ.

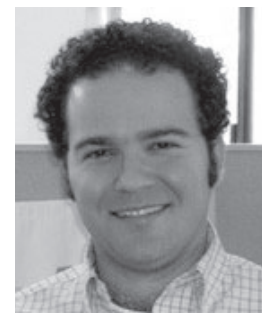

Scott Brenner BRAVE [non-member]

Scott is a founder and CTO of Baynote, Inc. Prior to Baynote, he was a postdoctoral scholar at Stanford University and served as lab manager for the CHIMe (Communication between Humans and Interactive Media) Lab. Scott is an inventor of six patents and co-author of over 25 publications in the areas of human-computer interaction and artificial intelligence. Scott is also a former Editor of the "International Journal of HumanComputer Studies" (Amsterdam: Elsevier) and co-author of "Wired for speech: How voice activates and advances the human-computer relationship" (Cambridge, MA: MIT Press). Scott received his Ph.D. in Human-Computer Interaction (Dept. of Communication), and B.S. in Computer Systems Engineering from Stanford University, and his Master's from the MIT Media Lab. 


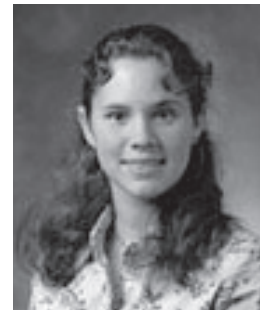

Heidy MALDONADO [non-member]

Heidy Maldonado is a $\mathrm{PhD}$ Candidate in Learning Sciences and Technology Design at Stanford University. She holds Bachelor and Master of Science degrees in Computer Science, all from Stanford University. She has published widely on topics related to her research interests, including computer supported collaborative learning and work; design and interaction with embodied expressive computer agents; mobile interface design; cross $^{-}$cultural interface design. Ms. Maldonado is thankful to have had the opportunity to collaborate on this project with OMRON Corporation.

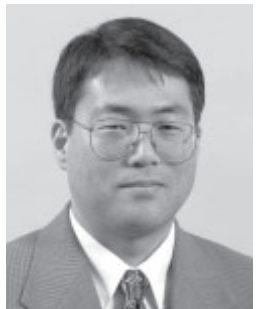

Yasunori MORISHIMA [non-member]

1996 年, コロラド大学大学院博士 課程修了. Ph.D. (認知心理学). Omron Advanced Systems (米国) Senior Researcherを経て，現在，国 際基督教大学教養学部 (心理学) 上級 准教授. 2002-2003年, スタンフォー ド大学客員研究員.

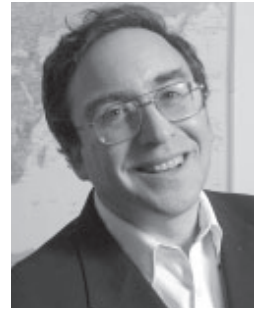

Clifford Ivar NASS [non-member]

Clifford Nass (Ph.D., Princeton University) is the Thomas M. Storke Professor at Stanford University, with appointments in Communication; Education; Science, Technology, and Society; Sociology; and Symbolic Systems. He is Director of the Communication between Humans and Interactive Media (CHIMe) Lab and co-director of CarLab at Stanford University. Nass is the author of two books, "The Media Equation" and "Wired for Speech," and over 100 papers in social aspects of human-technology interaction and statistical methodology. He is founder of the Computers Are Social Actors paradigm and has been involved in the design of over 200 interactive media products and services. 